
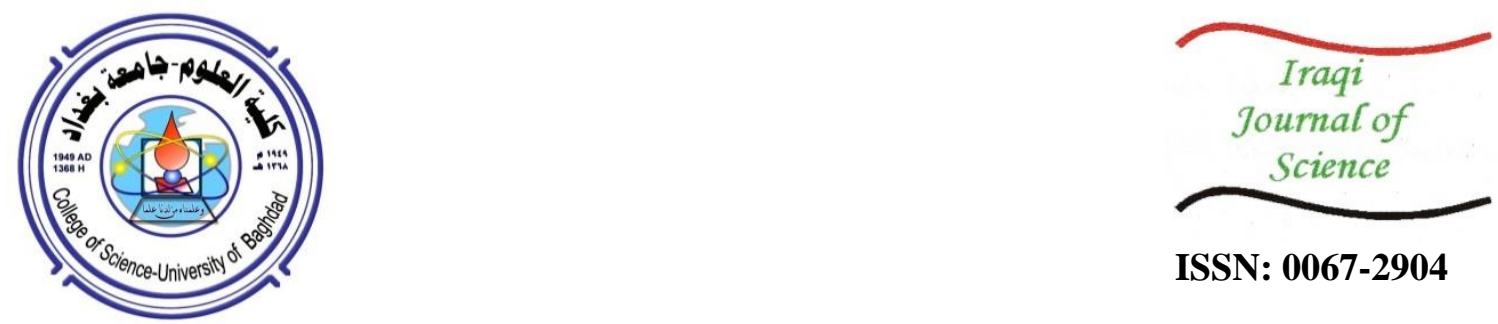

ISSN: 0067-2904

\title{
Enhancing the Accuracy of Health Care Internet of Medical Things in Real Time using CNNets
}

\author{
Israa Mishkhal ${ }^{1}$, Muntadher Khamees ${ }^{1}$, Hassan Hadi Saleh ${ }^{2}$ \\ ${ }^{1}$ Department of Computer Science, Science College, University of Diyala, Diyala, Iraq \\ ${ }^{2}$ Department of Computer Science, Faculty of physical education and sport silences, University of Diyala \\ Received: $12 / 12 / 2020$ \\ Accepted: 18/1/2021
}

\begin{abstract}
This paper presents an efficient system using a deep learning algorithm that recognizes daily activities and investigates the worst falling cases to save elders during daily life. This system is a physical activity recognition system based on the Internet of Medical Things (IoMT) and uses convolutional neural networks (CNNets) that learn features and classifiers automatically. The test data include the elderly who live alone. The performance of CNNets is compared against that of state-of-the-art methods, such as activity windowing, fixed sample windowing, timeweighted windowing, mutual information windowing, dynamic windowing, fixed time windowing, sequence prediction algorithm, and conditional random fields. The results indicate that CNNets are competitive with state-of-the-art methods, exhibiting enhanced IoMT accuracy of $98.37 \%$, which is the highest among the proposed solutions using the same dataset.
\end{abstract}

Keywords: Internet of Medical Things (IoMT), IoT, Deep learning, CNNets, Network sensors, RFID.

\section{تحسين دقة اداء إنترنت الأجهزة الطبية للرعاية الصحية في الوقت الفعلي باستخدام خوارزمية CNNets}

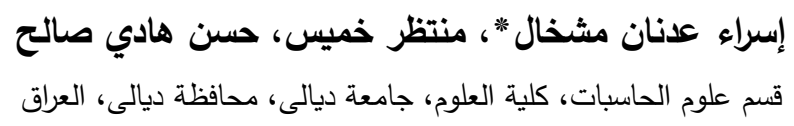

يناقش هذا البحث نظامًا فعالًا باستخدام احد خوارزميات التعلم العميق التي تتعرف على الأنشطة اليومية

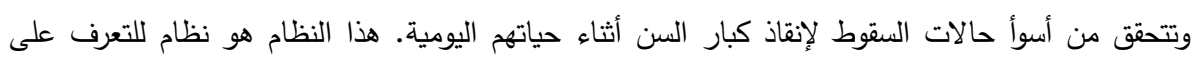

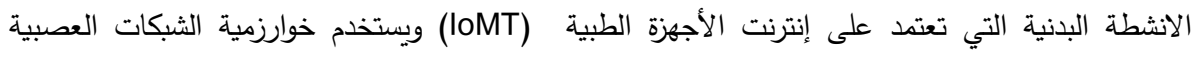

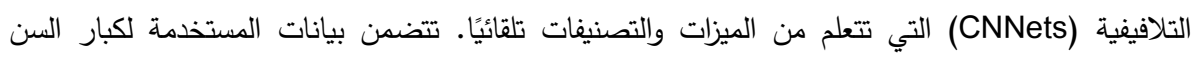

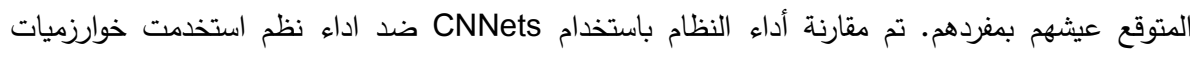

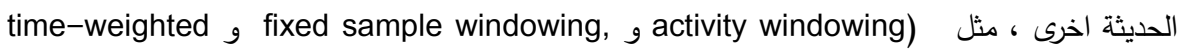
fixed time, dynamic windowing g mutual information windowing, windowing 


$$
\begin{aligned}
& \text { conditional random fields g the sequence prediction algorithm, windowing } \\
& \text { النتائج إلى أن CNNets قادرة على المنافسة مع أحدث الأساليب المستخدمة، حيث أظهر دقة loMT محسّنة } \\
& \text { بنسبة 98.37٪، وهذا اعلى نسبة بين الحلول المقترحة التي اختبرت على نفس البيانات المستخدمة بهذه } \\
& \text { الدراسة. }
\end{aligned}
$$

\section{Introduction}

The Internet of Things (IoT) is a challenging concept that has many issues. About 18.2 billion smart devices are connected by the Internet [1]. Therefore, the IoT covers all categories across the globe. The IoT is the internetworking of intelligent machines in different domains to transfer data between devices without human assistance [2]. Furthermore, it helps improving people's lives, managing people's health, and providing help in severe cases via smart devices [3].

Moreover, the IoT is a complex system that enables data exchange depending on the application. For instance, in small applications, the IoT system considers a unique object that is connected to the Internet and static data. Data collected from sensors can be used anywhere and anytime [4]. Unlike broad applications, the IoT involves numerous things connected to the Internet to provide sophisticated service. This process may or may not involve human intervention and is controlled through intelligent interfaces for data mining, communication, and actuation [4].

The IoT has an impact on the medical field. About $40 \%$ of IoT devices in the medical domain, particularly in the healthcare field, were expected to be connected by 2020 , reaching a value of up to 117 billion dollars in the market [5]. Therefore, the Internet of Medical Things (IoMT) is a part of the IoT, as it connects medical devices to a health system through the Internet, e.g. through a cloud system [6]. The IoMT has been used for monitoring activities, such as smart devices for mobile health, remote monitoring of chronic illnesses, and tracking of medication orders [7, 8].

Information and communication technology (ICT) plays a significant role in the IoMT, particularly through products by Apple, Google, Amazon, and Cisco. In 2014, Novartis cooperated with Google to produce sensor technology, including a wearable device to measure blood glucose levels and the smart lens [9]. Amazon cooperated with Merck and Luminary Labs to find the perfect way of monitoring diabetes on the basis of voice-enabled solutions in 2017 [10]. The IoMT plays a significant role in recognizing the daily activities of people [11], which helps detect early indicators of emergency cases that elderly patients may encounter, such as heart failure and fall conditions.

Sensors are an essential part of the IoMT. Without sensors, data cannot be collected from the environment and IoMT applications are impossible to develop. Network sensors are a collection of antennas working together. Wearable wireless identification and sensing platform (W2ISP) devices worn by healthy participants are connected to several radiofrequency identification (RFID) sensors. They are distributed in a particular setting, such as a clinic, to collect data on daily activities. These network sensors have three RFID antennas and one W2ISP. Therefore, IoMT technology helps people prepare for the worst cases that may happen to them. Furthermore, doctors can obtain rich information that can help them treat their patients [12].

\subsection{Radio Frequency Identification}

RFID is a rapidly growing technique that uses radio waves to collect and transmit information. Its uses and advantages in health care have been investigated in recent years. The use of RFID tags in an indoor tracking system for older adults was proposed in 2011 [13]. This system used the information obtained from the environment by tracking the signal 
strength from the RFID sensor. Furthermore, it exhibits good accuracy in the U-Healthcare system in terms of the real-time recognition of older adults' location.

RFID is used in some hospitals to detect a patient's location for emergency cases. For example, a Belgian hospital implemented a real-time locating system based on the RFID antenna [14]. RFID is also used in the access control system of healthcare. This system aims to mitigate safety and security risks in shady areas of the hospital because of external threat or human error [15]. Without human interference, the RFID can collect information automatically. Unlike barcode scanning, RFID does not require line-of-sight for readers to capture information from tags [16].

Typically, a RFID scheme comprises a transponder and a software application for the transponder [17]. Active or passive transponders can be used. An active transponder has its own energy source, whereas a static transponder is used as an energy source by the transponder reader. The transponder reader uses radio frequency signals to obtain data from the tag, including identification values, information encrypted in the tag, and its location [13]. The data gathered from the reader of the transponder are sent to a database mounted on a server via a local area network (LAN). Users can obtain information from server-installed software. The use of RFID has many advantages to patient safety, patient monitoring, patient care efficiency, and supplier satisfaction in the health industry. Research shows that RFID can improve safety for patients [18].

\subsection{Wireless Identification and Sensing Platform (WISP)}

WISP is an RFID device that promotes tracking and computation, that is, a microcontroller driven by radio frequency energy [19]. As a passive RFID, WISP is read and powered by a standard RFID, recharging the battery from the radio signals emitted to the reader. A WISP is a normal EPC gen1 or gen2 tag for a RFID reader, but the harvested energy operates a 16-bit general-purpose microcontroller inside the WISP. The microcontroller can conduct a range of computing functions, including sampling of detectors and reporting sensor information back to the RFID viewer. WISPs are constructed with light sensors, temperature sensors, and strain gauges. WISPs can refer to flash and carry out cryptographic computations [20].

\subsection{Wearable Wireless Identification and Sensing Platform (W2ISP)}

Wearable WISP (W2ISP) devices are designed by [21]. As shown in Figure 1, W2ISP is a small, easy-to-maintain device that is wearable over clothing [22-24]. Thus, older patients can wear them for experiments. In 2019, a new wearable sensor system was built to increase elders' confidence in sensing technology [23]. The RFID circuit board and tracking unit are included in the W2ISP, with a versatile antenna (the sensor hereinafter) for the convenience of patients and a washable RIPSTOP silver-coated nylon to insulate the machine and the person (see Figure 1). With a supply voltage of $1.8 \mathrm{~V}$ and a typical output sensitivity of $300 \mathrm{mV} / \mathrm{g}$, the three-axis (ADXL330) accelerometer has a minimum full-scale range of $\pm 3 \mathrm{~g}$ and a low energy demand of $180 \mu \mathrm{A}$. MSP430F 2132 is a low-powered 16-bit flash unit that contains a 10-bit samples-per-second analog-to-digital converter [25]. The W2ISP can provide information related to a person's orientation and activities by combining the automatic and precise identification capabilities of RFID tags [23]. 


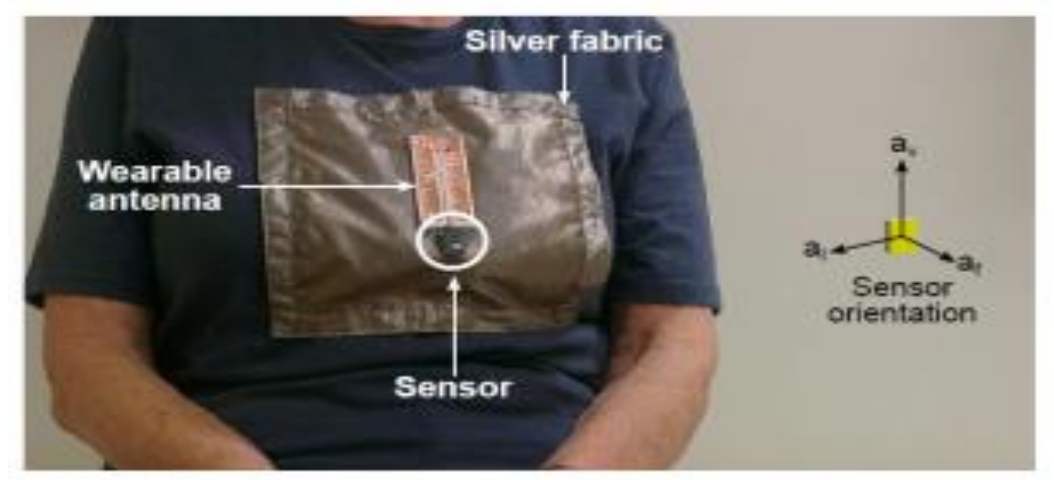

Figure1-W2ISP [23]

\subsection{Deep learning}

Deep learning is a category of machine learning (ML) algorithms that use several layers to draw from raw input characteristics gradually. Deep learning differs from conventional ML in how raw information is used for learning. Deep learners can learn data representations with multiple abstraction levels through computer models consisting of several processing levels based on neural networks. The main distinctions between deep learning and traditional artificial neural networks are the number of hidden layers, the links, and the ability to understand significant input abstractions. Deep learning algorithms have achieved excellent accuracy for many industries that test them in their research. Google, Facebook, Netflix, Apple, IBM, and Microsoft have used in-depth knowledge to improve their works [26]. These methodologies have also been used to identify health issues and complex problems; for example, Alzheimer's [27], activity recognition [28], and handwriting numeric recognition [29]. Thus, deep learning algorithms are a technique for learning machines and finding another feature of data inputs [30].

\section{Literature Review}

Recently, health issues have become important in the IoT domains. Therefore, researchers have begun their studies to find the best solution to improve the IoMT field. United Nations reports show that more than $30 \%$ of the population of 64 countries are old adults who live with chronic diseases [31]. Falling is the worst problem for them, which is why doctors advise fall prevention measures in hospitals or homes [32]. Moreover, an earlier work [33] showed that approximately 26 patient deaths, 530 hip fractures, and 1,000 elders have had a type of fracture due to falling. Falls could have other psychological consequences, such as fear of falling, depression, lack of confidence, and anxiety. Thus, researchers are continuously looking for ways to help the elderly, because they are starting to live alone, according to studies [34-37].

A basic design that combines the 11073 IEEE Service/DIM and CoAP to produce a device for healthcare was introduced in 2016 [38]. The best comparison of performance between 11073 DIM and HTTP was presented and evaluated with HTTP and CoAP in terms of packet abundance in a single transaction, syntax, and packet loss rate by using JSON and XML. In another study [39], the authors state that XML is not better than JSON. By using bodyweight, a technique to recognize the patient posture that exerts pressure on a specially designed pillow was proposed [35]. To enhance the IoMT, researchers used many types of sensors to monitor activities and collect data [39]. Some types of sensors used for the first time in monitoring include RFID and batteryless sensors, which utilize lightweight RFID, an exclusive technology [40]. These sensors are acceptable for elders [22]. Researchers have also started to use a network of sensors to increase the data collection accuracy [35, 41, 42]. 


\section{Methodology}

The collecting data room has two types of sensors, as shown in Figure 2, namely, W2ISP and the RFID reader. This network of sensors was developed in earlier investigations [22, 43-45]. W2ISP involves ADXL330, which is the triaxial accelerometer antenna. The processor of W2ISP is MSP430F2132, which is a microprocessor that has a silver fabric that can isolate a patient [46]. The RFID antenna has a UHF-based RFID reader (IPJ-REV-R420-GX11M) that runs in the frequency band of $920-926 \mathrm{MHz}$ and a circularly polarized sensor, which is Laird S9028PCLJ [47]. The RFID sensor is lightweight and small and has a self-charging battery. Based on the energy in the electromagnetic field, the antenna can recharge [47]. Furthermore, the RFID antenna (ISO 18000-6C) follows the air interface protocol, which is used to connect the RFID sensor to the W2ISP [48]. This connection helps collect data from participants. A single RFID sensor can simultaneously connect with many W2ISP sensors.

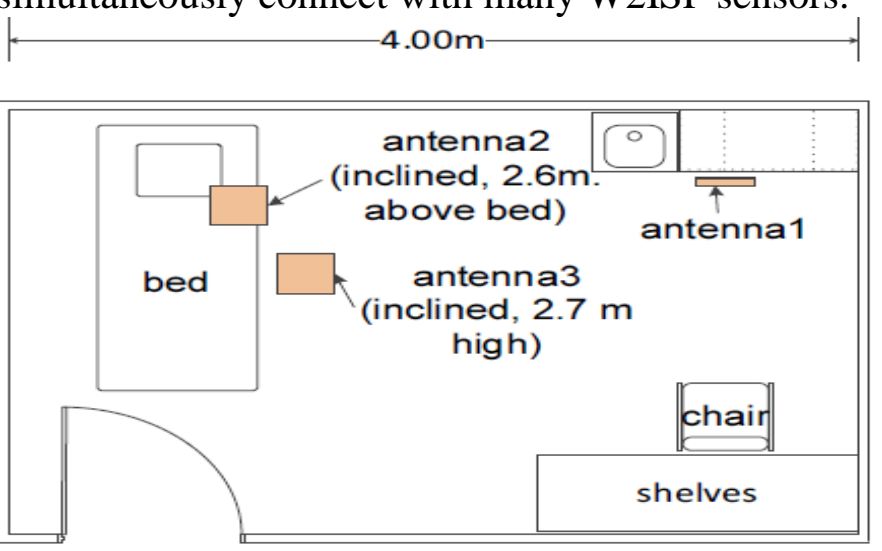

Figure 2-Configuration used for trials

The first type is to measure the frontal, vertical, and lateral accelerations along the axes and the acceleration of motion from a person (Figure 3). The second type is to measure the strength signal of the W2ISP and then to perform signal activation. According to literature $[22,24,38,43,45]$, the link distance between RFID and W2ISP is represented as the strength of the W2ISP signal (RSSI), and recorded the ID (as aID) that obtains a specific read from the W2ISP. Therefore, the data collected using these network sensors show some features, such as af, av, al, RSSI, F, $\phi$, aID, T, and S (Table 1 and Figure 4). The maximum distance that these sensors can measure is $4 \mathrm{~m}$ [25].

Table 1-Description of the input features for the convolutional 1D layer.

\begin{tabular}{|c|c|}
\hline Feature & Description \\
\hline al & lateral acceleration \\
\hline af & frontal acceleration \\
\hline av & vertical acceleration \\
\hline RSSI & received signal strength indicator \\
\hline F & frequency \\
\hline aID & receiving antenna identification \\
\hline S & antenna \\
\hline T & time \\
\hline$\phi$ & phase \\
\hline
\end{tabular}

The data collected from the ambulatory movement of elderly participants were presented and used [22, 43-45]. Fourteen healthy old volunteers (4 males and 10 females) with age range of 66-86 years participated in this experiment. Each volunteer wore the W2ISP sensor at a high 
level on his/her chest (Figure 1). The sensor captures the movement signals necessary to recognize high fall risk activities. The four types of activities selected for this experiment are ambulation, lying, sitting on a bed, and sitting on a chair. As shown in the Figure 2, the dataset was collected from the clinical room at the Elizabeth Hospital. This room has three RFID antennas distributed inside it in different locations; two are at the ceiling level, and the other is on the wall. These sensors focus on areas that patients often use. These error signals could be produced by obstructions, which could affect collecting data from the RFID and W2ISP sensors. These error signals are avoided by placing the antenna in different levels inside the room [43].

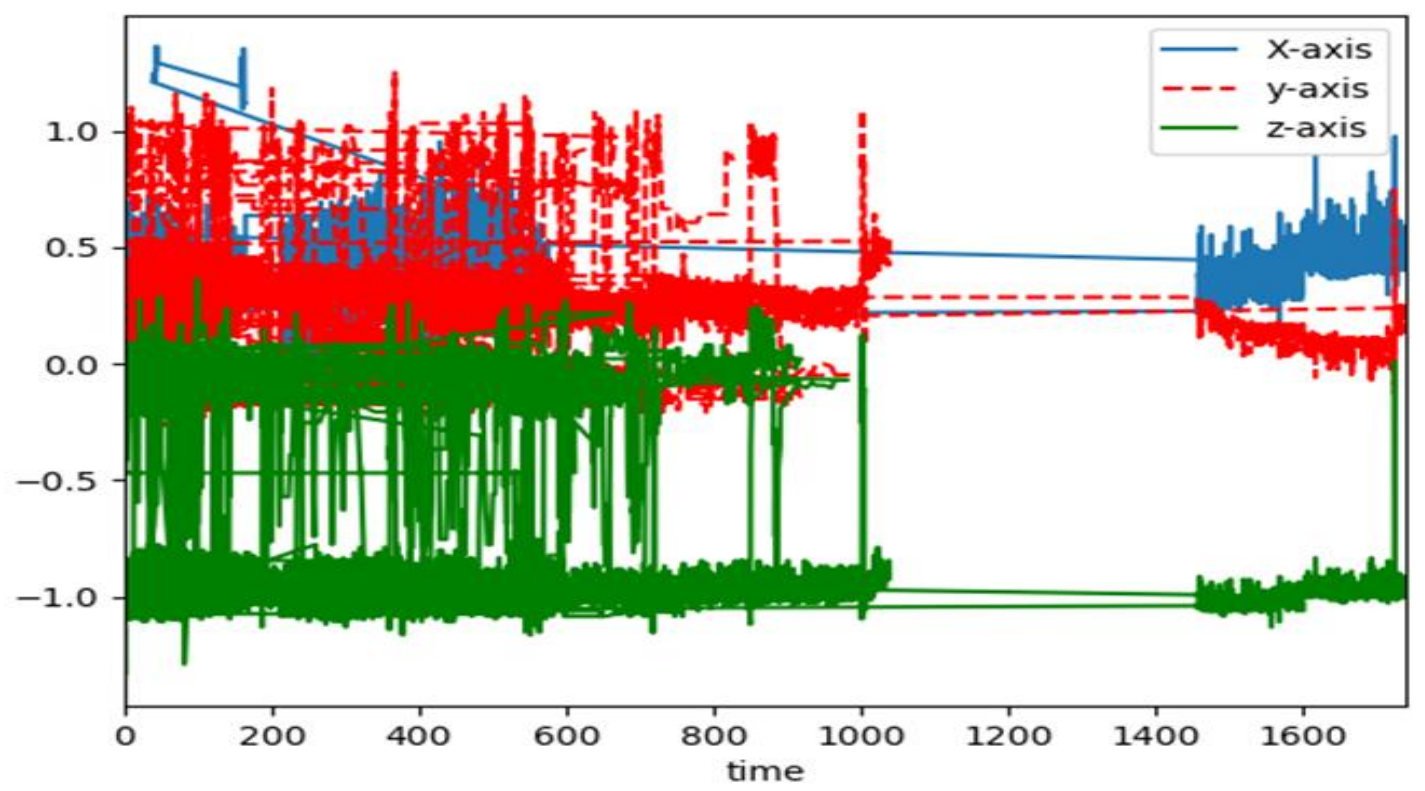

Figure 3-Three triaxial embedded W2ISP with thresholds used for recognizing lying activity.

The proposed system is designed to predict the worst fall cases that are considered of high health risks to the elderly [49]. This system is based on CNNets' methodology. It was used to predict these cases by learning about the four chosen types of daily activities.

CNNets have three main layers, namely, the input, hidden, and output layers. They also have activation layers for modifying the signals produced by the previous layers, such as Softmax, ReLU, and Tanh. The activation layers use the following equations (1.1) and (1.2):

$$
\begin{gathered}
\operatorname{Softmax}(X)=\mathrm{e}^{\mathrm{Xi} /} \sum_{\mathrm{k}=1}^{\mathrm{k}} \mathrm{e}^{\mathrm{Xk}} \quad \text { for } \mathrm{i}=1 \ldots \ldots \mathrm{k} \\
\operatorname{ReLU}(\mathrm{X})=\left\{\begin{array}{l}
0 \text { for } \mathrm{X}<0 \\
\mathrm{X} \text { for } \mathrm{X} \geq 0
\end{array}\right.
\end{gathered}
$$

$\mathrm{X}$ is an input data of previous layers. 


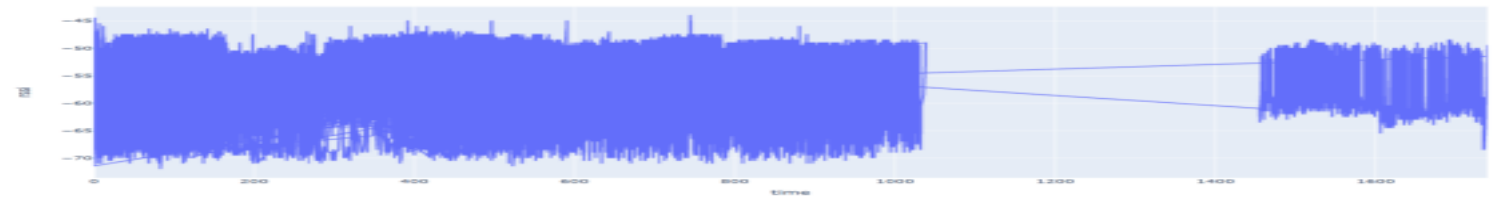

(a)

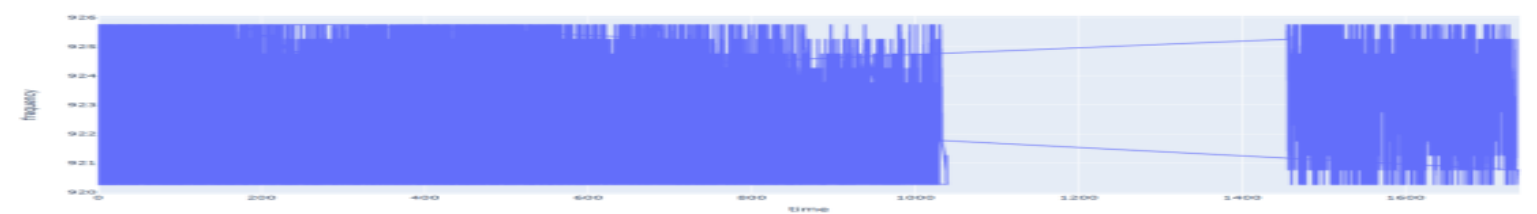

(b)

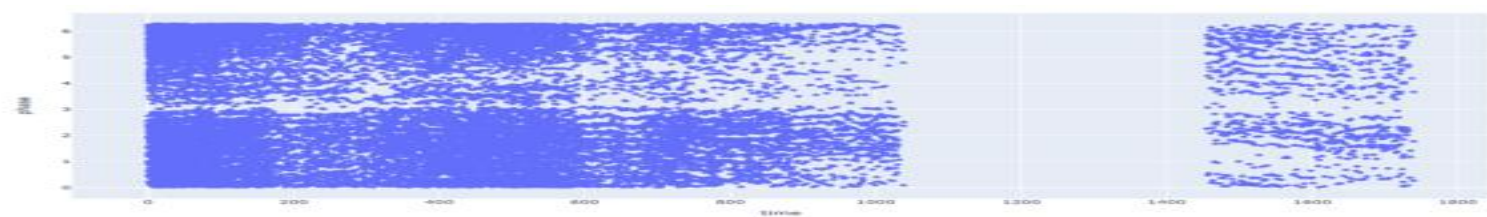

(c)

Figure 4-The $x$-axis shows real time while $y$-axis represents significant features. a) RSSI, b) F, c) phase $\phi$

This system consists of more than one input layer; namely, convolution and pooling layers (see Figure 5). As shown in Table 1, the first layer takes (af, av, al, RSSI, F, $\phi$, aID, T, and S) features and multiples them by an individual filter's size $(3 \times 3)$ according to the experiment described elsewhere [28]. As shown in Equation (1.3), the convolution layer has a set of local filters that can capture the small parts of the input data and a pooling layer that preserves the critical features of the previous output layer. The fully connected layer, which is the last layer of the CNNets, combines all the input features inferred from the previous layers for classification and recognizes the four types of daily activities performed by the participants during the data collection stage, thus helping detect the dangers of falling in an effort to reduce the death rate.

$$
Y_{i}^{\mathrm{x}, \mathrm{d}}=\mathrm{V}\left(\mathrm{bij}+\sum_{\mathrm{m}} \sum_{\mathrm{p}=0}^{\mathrm{pi}-1} \mathrm{~K}_{\mathrm{ijm}}^{\mathrm{p}} \mathrm{Y}_{(\mathrm{i}-1) \mathrm{m}}^{\mathrm{x}+\mathrm{p}, \mathrm{d}}\right)
$$

$\forall \mathrm{d}=1, \ldots, \mathrm{D}$

$Y_{i j}^{x, d}$ : Output of the convolution layer.

$V$ : Hyperbolic tangent function.

bij: Bias for a feature map.

$m$ : Indexes over the set of the feature map.

$K_{i j m}^{p}$ : Value at the position $p$ of kernel. 


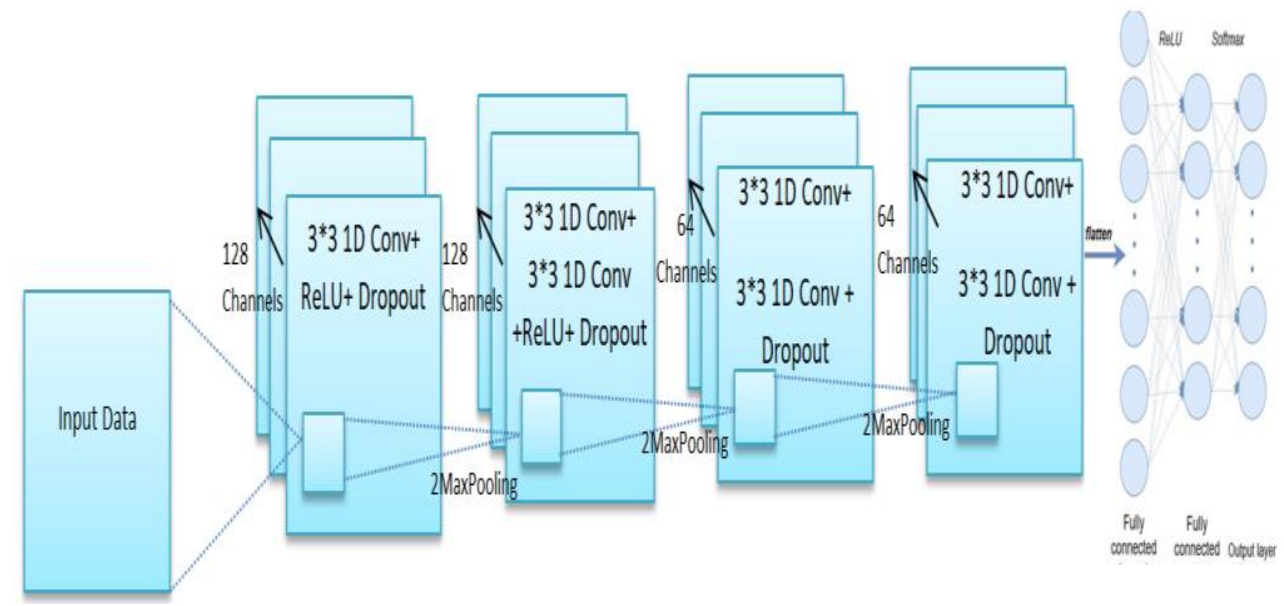

Figure 5-CNNets structure

\section{Results and Analysis}

The data adopted from other studies are representative of elders who live alone [22, 43-45]. The healthy participants performed four types of high fall risk activities, namely, sitting on a bed, lying, ambulation, and sitting on a chair, inside a clinic room provided with three RFID sensors installed at different places (see Section 3).

The experimental results depend on some effective factors, which include the distance between the sensors inside the clinic room and the sensor held by the participator, body motion, and the multipath inside the testing room. The collected data were divided into training $(70 \%)$ and testing $(30 \%)$ data according to the numbers of training epochs. The experiment was performed using Python on a computer with 8 GB RAM, $1867 \mathrm{MHz}$ DDR3 memory, and an EI Capitan ver. 10.11.6 operating system. The programming language used to implement the proposed system has a specialized library called Keras, which compensates for the system network's inefficiencies. The machine used in this experiment could have imposed limitations; therefore, the proposed solution took more than 15 minutes to implement. As a result, it achieved higher accuracy than other methodologies, as shown in (Figure 6). The accuracy was calculated as $\mathrm{P}=\mathrm{TP} /(\mathrm{TP}+\mathrm{TN})$ and trained on 6794 of 15851 samples from the dataset with 8 features in 300 epochs. Also, the lying down activity achieved the highest prediction accuracy than other types of activities, as shown in (Figure 7), since the participators were most the time of the experiment lying on beds. 


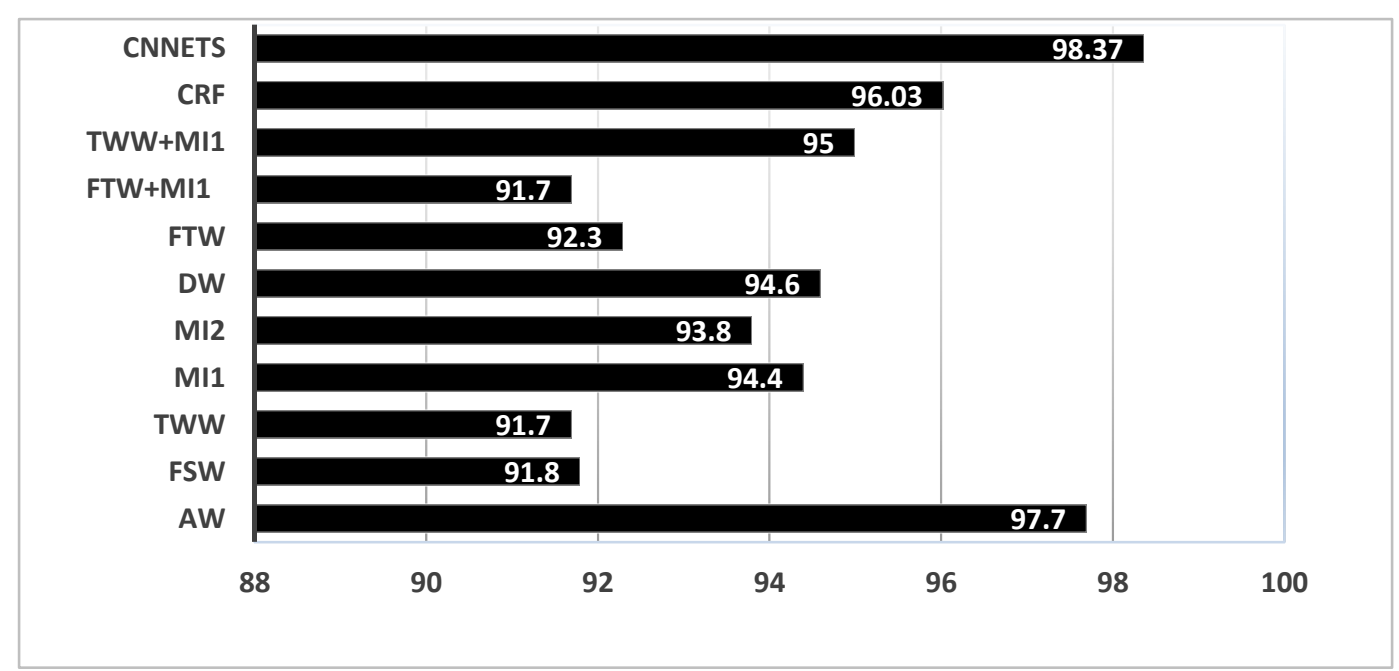

Figure 6-Methodology comparison

The results of the newly proposed method are better than those of previous methods. Table 2 summarizes the results of previous works used to solve fall issues. The proposed approach has an accuracy of about $98.37 \%$, which is the highest compared with other approaches, since it used IoMT sensors to collect the data and chose four types of essential daily activities. Furthermore, it selected 8 features (af, av, al, RSSI, F, $\phi$, aID, T, and S) that deem of the critical features to enhance the accuracy rate using CNNets that recognize and detect the selected activities.

Table 2-Classification accuracy

\begin{tabular}{|c|c|}
\hline Method & Accuracy \\
\hline SVM & 96.1 \\
\hline CRFs & 93.4 \\
\hline AW & 97.7 \\
\hline FSW & 91.8 \\
\hline TWW & 91.7 \\
\hline MI1 & 94.4 \\
\hline MI2 & 93.8 \\
\hline DW & 94.6 \\
\hline FTW & 92.3 \\
\hline FTW+MI1 & 91.7 \\
\hline TWW+MI1 & 95.0 \\
\hline CRF & 96.03 \\
\hline CNNets & 98.37 \\
\hline
\end{tabular}

Table 2 shows that the activity windowing (AW) method tests only 17 samples for sitting-inchair. This activity was overlooked and resulted in a false negative (FN) outcome, thereby affecting all other activities' metrics. This methodology achieved an accuracy of $97.7 \%$. The fixed sample windowing (FSW) recorded 91.8\% accuracy, which is lower than that of the AM method, because the collected data depended only on lying and sitting-in-bed movements that cause mutual errors, such as those observed by the FN result of sitting-in-bed and the false positives (FPs) in ambulating. These errors were minimized in the second approach [45]. Given the mutual error between lying and sitting in bed, the time weighted windowing 
(TWW) achieved about $91.7 \%$ accuracy, which is lower than that achieved by the previous solutions. The sequence prediction algorithm's (SVM) accuracy for three labels (sitting on the bed, lying in bed, and out-bed) is $96.1 \%$ [5]. The time for the actual bed egress activity is considered zero. The authors defined true position (TP) as the out-bed movement, while the in-bed position was defined as FP. Moreover, the other missed bed-egress movements were defined as FN. The accuracy for the presented solution was calculated as $\mathrm{P}=\mathrm{TP} /(\mathrm{TP}+\mathrm{FP})$. Conditional random fields (CRFs) were used to enhance the label prediction of each daily activity and recognize whether a patient moved from the bed or not. This methodology achieved about $93.4 \%$ accuracy. The CRFs always depended on the sequence of activities [22]. Then, the authors proposed a new system using the CRF. The system predicted three types of daily activities: lying in bed, sitting on the bed, and out of bed. The FPs were fewer than those of the previous methods [22]. The previous methods described a higher number of TPs. The CRF achieved 95.03\%, which is higher than the CRFs with fewer false alarms [43]. The combination cases, TWW+MI1 and TFW+MI1, achieved accuracies of $95.0 \%$ and $91.7 \%$, respectively. The accuracy of TWW+MI1 was higher than that of the other combination case because it has rich extended information. MI1 and MI2 achieved accuracies of $94.4 \%$ and $93.8 \%$, respectively. This contrast in the result could be attributed to the transition values of the two sensors placed on top of the bed. Most of the recorded data were in-bed and around-bed [45]. Furthermore, the DW and FTW methods achieved accuracies of $94.6 \%$ and $91.8 \%$, respectively. The DW result is higher than the FTW result because it has extra features.

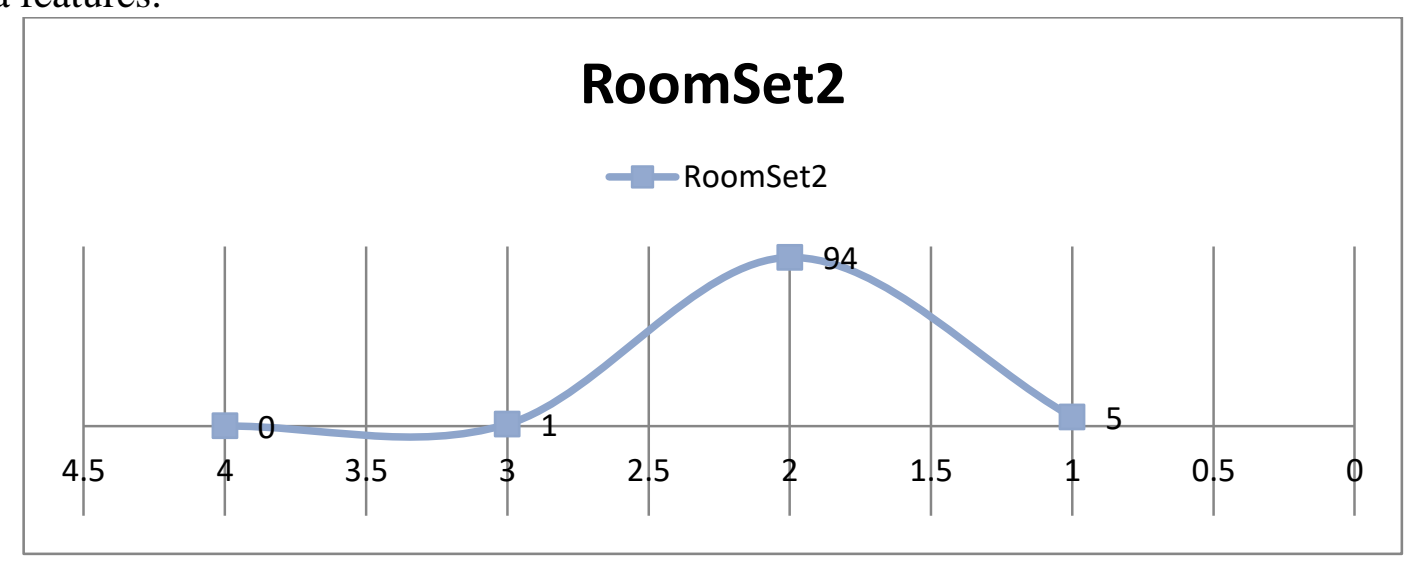

Figure 7-Accuracy rate for four types of daily activities

Some benefits were derived from the proposed approach. The IoMT medical sensors are designed to be flexible. They are made from foam and conductive fabric, which allows the elders to move freely. The elders can also remove the W2ISP sensor from their clothes directly. Some IoMT medical sensors are batteryless sensors and use lightweight RFID [40]. Thus, elderly people find these antennas acceptable. Moreover, the performance of this system affects the length of the subsequent comparison with traditional CNNets. Thus, the sliding window size needs to be adjusted for the prediction stage.

\section{Conclusions}

The IoMT remodels health services and helps manage people's health requirements. Many medical devices are being used to monitor people's health parameters, such as blood pressure and calories burned. The best domain of the IoMT is the remote health monitoring or recognition system, which is used to obtain data from anywhere. By using such services, doctors can quickly determine the worst cases that may happen to people. Monitoring the elderly has become essential in reducing the death rate. In this work, data were collected by designing a clinic room with a network of antennae for older adults at the Elizabeth Hospital. 
With the use of the air interface protocol, W2ISP was connected to three RFIDs distributed inside the clinical room. The data collection focused on four activities, namely, sitting on a bed, lying down, ambulating, and sitting on a chair, because of the limited movements of the elderly. CNNets were proposed to recognize and predict falls and to improve the accuracy of IoMT. Our proposed system achieves $98.37 \%$ accuracy. This accuracy is higher than that of the other systems.

\section{References}

[1] Internet of Things (IoT): Number of Connected Devices Worldwide From 2012 to 2020 (in billions). [Online]. Available: https://www.statista.com/statistics/471264/iot-number-ofconnected- devices-worldwide/

[2] Gubbi, Jayavardhana, et al. "Internet of Things (IoT): A vision, architectural elements, and future directions." Future generation computer systems 29.7 (2013): 1645-1660.

[3] Andriopoulou, Foteini, Tasos Dagiuklas, and Theofanis Orphanoudakis. "Integrating IoT and fog computing for healthcare service delivery." Components and services for IoT platforms. Springer, Cham, 2017. 213-232.

[4] Minerva, Roberto, Abyi Biru, and Domenico Rotondi. "Towards a definition of the Internet of Things (IoT)." IEEE Internet Initiative 1.1 (2015): 1-86.

[5] Bauer, Harald, Mark Patel, and Jan Veira. "The Internet of Things: Sizing up the opportunity." Retrieved from: McKinsey at http://www. mckinsey. com/insights/high_tech_telecoms_internet/the_internet_of_t hings_sizing_up_the_opportunity (2014).

[6] Basatneh, Rami, Bijan Najafi, and David G. Armstrong. "Health sensors, smart home devices, and the internet of medical things: an opportunity for dramatic improvement in care for the lower extremity complications of diabetes." Journal of diabetes science and technology 12.3 (2018): 577-586.

[7] Armstrong, David G., Bijan Najafi, and Mohsen Shahinpoor. "Potential applications of smart multifunctional wearable materials to gerontology." Gerontology 63.3 (2017): 287-298.

[8] Miller, John D., Bijan Najafi, and David G. Armstrong. "Current standards and advances in diabetic ulcer prevention and elderly fall prevention using wearable technology." Current Geriatrics Reports 4.3 (2015): 249-256.

[9] Senior, Melanie. "Novartis signs up for Google smart lens." Nature biotechnology 32.9 (2014): 856-857.

[10] Coombs, Bertha. "How Alexa's best skill could be as a home health-care assistant." CNBC. August 9 (2017).

[11] Liu, Xi, et al. "Human daily activity recognition for healthcare using wearable and visual sensing data." 2016 IEEE International Conference on Healthcare Informatics (ICHI). IEEE, 2016.

[12] Opazo Basaez, Marco, et al. "Smart healthcare services: A patient oriented cloud computing solution." (2014).

[13] Yao, Wen, Chao-Hsien Chu, and Zang Li. "The use of RFID in healthcare: Benefits and barriers." 2010 IEEE International Conference on RFID-Technology and Applications. IEEE, 2010.

[14] Gholamhosseini, Leila, Farahnaz Sadoughi, and Aliasghar Safaei. "Hospital real-time location system (A practical approach in healthcare): A narrative review article." Iranian journal of public health 48.4 (2019): 593.

[15] Figueroa, Santiago, Javier Añorga, and Saioa Arrizabalaga. "An attribute-based access control model in RFID systems based on blockchain decentralized applications for healthcare environments." Computers 8.3 (2019): 57.

[16] Lahtela, Antti. "A short overview of the RFID technology in healthcare." 2009 Fourth International Conference on Systems and Networks Communications. IEEE, 2009.

[17] Idachaba, F. E., and U. S. Tommy. "RFID laptop monitoring and management system." (2014).

[18] Paaske, Stephanie, et al. "The benefits and barriers to RFID technology in healthcare." On-Line Journal of Nursing Informatics 21.2 (2017). 
[19] Van der Togt, Remko, Piet JM Bakker, and Monique WM Jaspers. "A framework for performance and data quality assessment of Radio Frequency IDentification (RFID) systems in health care settings." Journal of Biomedical Informatics 44.2 (2011): 372-383.

[20] Liao, Lingxia, Guohuan Lou, and Min Chen. "An Integrated RFID and sensor system for emergency handling in underground coal mines environments." International Conference on Ad Hoc Networks. Springer, Berlin, Heidelberg, 2009.

[21] Sample, Alanson P., et al. "Design of a passively-powered, programmable sensing platform for UHF RFID systems." 2007 IEEE international Conference on RFID. IEEE, 2007.

[22] Torres, Roberto L. Shinmoto, et al. "Sensor enabled wearable RFID technology for mitigating the risk of falls near beds." 2013 IEEE international conference on RFID (RFID). IEEE, 2013.

[23] Wickramasinghe, Asanga, and Damith C. Ranasinghe. "Recognising activities in real time using body worn passive sensors with sparse data streams: To interpolate or not to interpolate?." Proceedings of the 12th EAI international conference on mobile and ubiquitous systems: computing, networking and services on 12th EAI international conference on mobile and ubiquitous systems: computing, networking and services. 2016.

[24] Jayatilaka, Asangi, et al. "Designing batteryless wearables for hospitalized older people." Proceedings of the 23rd International Symposium on Wearable Computers. 2019.

[25] Wickramasinghe, Asanga, Damith C. Ranasinghe, and Alanson P. Sample. "Windware: Supporting ubiquitous computing with passive sensor enabled rfid." 2014 IEEE International Conference on RFID (IEEE RFID). IEEE, 2014.

[26] Dolata, Ulrich. Apple, Amazon, Google, Facebook, Microsoft: Market concentrationcompetition-innovation strategies. No. 2017-01. SOI Discussion Paper, 2017.

[27] Sarraf, Saman, and Ghassem Tofighi. "Classification of alzheimer's disease using fmri data and deep learning convolutional neural networks." arXiv preprint arXiv:1603.08631 (2016).

[28] Mishkhal, Israa Adnan. "Human activity recognition based on accelerometer and gyroscope sensors." (2017).

[29] Ashiquzzaman, Akm, and Abdul Kawsar Tushar. "Handwritten Arabic numeral recognition using deep learning neural networks." 2017 IEEE International Conference on Imaging, Vision \& Pattern Recognition (icIVPR). IEEE, 2017.

[30] Dertat, Arden. "Applied deep learning-part 4: Convolutional neural networks." Towards Data Science (2017).

[31] Fund, UN Populations, and H. A. International. "Ageing in the twenty-first century: a celebration and a challenge." (2012).

[32] Cameron, Ian D., et al. "Interventions for preventing falls in older people in care facilities and hospitals." Cochrane database of systematic reviews 9 (2018).

[33] Oliver, David, Frances Healey, and Terry P. Haines. "Preventing falls and fall-related injuries in hospitals." Clinics in geriatric medicine 26.4 (2010): 645-692.

[34] Mubashir, Muhammad, Ling Shao, and Luke Seed. "A survey on fall detection: Principles and approaches." Neurocomputing 100 (2013): 144-152.

[35] Najafi, Bijan, et al. "Ambulatory system for human motion analysis using a kinematic sensor: monitoring of daily physical activity in the elderly." IEEE Transactions on biomedical Engineering 50.6 (2003): 711-723.

[36] Wei, Bo, et al. "Radio-based device-free activity recognition with radio frequency interference." Proceedings of the 14th International Conference on Information Processing in Sensor Networks. 2015.

[37] Wright, John, et al. "Robust face recognition via sparse representation." IEEE transactions on pattern analysis and machine intelligence 31.2 (2008): 210-227.

[38] Ge, Shu-yuan, et al. "Design and implementation of interoperable IoT healthcare system based on international standards." 2016 13th IEEE annual consumer communications \& networking conference (CCNC). IEEE, 2016.

[39] Kosse, Nienke M., et al. "Sensor technologies aiming at fall prevention in institutionalized old adults: a synthesis of current knowledge." International journal of medical informatics 82.9 (2013): 743-752. 
[40] Ranasinghe, D. C., et al. "Low cost and batteryless sensor-enabled radio frequency identification tag based approaches to identify patient bed entry and exit posture transitions." Gait \& posture 39.1 (2014): 118-123.

[41] Bao, Ling, and Stephen S. Intille. "Activity recognition from user-annotated acceleration data." International conference on pervasive computing. Springer, Berlin, Heidelberg, 2004.

[42] Liu, S., et al. "Multisensor data fusion for physical activity assessment". IEEE Transactions on Biomedical Engineering, 59.3(2011): 687-696.

[43] Wickramasinghe, Asanga, et al. "Sequence learning with passive RFID sensors for real-time bedegress recognition in older people." IEEE journal of biomedical and health informatics 21.4 (2016): 917-929.

[44] Shinmoto Torres, Roberto L., et al. "A battery-less and wireless wearable sensor system for identifying bed and chair exits in a pilot trial in hospitalized older people." PloS one 12.10 (2017): e0185670.

[45] Torres, Roberto Luis Shinmoto, Damith C. Ranasinghe, and Qinfeng Shi. "Evaluation of wearable sensor tag data segmentation approaches for real time activity classification in elderly." International conference on mobile and ubiquitous systems: computing, networking, and services. Springer, Cham, 2013.

[46] Sample, Alanson P., et al. "Design of an RFID-based battery-free programmable sensing platform." IEEE transactions on instrumentation and measurement 57.11 (2008): 2608-2615.

[47] Ranasinghe, Damith C., M. Sheng, and Sherali Zeadally. "Unique radio innovation for the 21 st Century: building scalable and global RFID networks." (2010).

[48] EPCglobal, G. S. Inc.,"EPCTM Radio-Frequency Identity Protocols Class-1 Generation-2 UHF RFID Protocol for Communications at 860MHz-960MHz Version 2.0. 1,”. EPCGlobal Inc., Tech. Rep., April 2015.[Online]. Available: http://www. gs1. org/epcrfid/epc-rfid-uhf-air-interfaceprotocol/2-0-1, 2015.

[49] Su, Yang, Asanga Wickramasinghe, and Damith C. Ranasinghe. "Investigating sensor data retrieval schemes for multi-sensor passive RFID tags." 2015 IEEE International Conference on RFID (RFID). IEEE, 2015. 